\title{
O-GIcNAcylation of the human epidermal growth factor receptor
}

\author{
Silviya R. Stateva and Antonio Villalobo* \\ Department of Cancer Biology, Instituto de Investigaciones Biomédicas, \\ Consejo Superior de Investigaciones Científicas and Universidad Autónoma de \\ Madrid \\ c/ Arturo Duperier 4, E-28029 Madrid, Spain
}

\begin{abstract}
*To whom correspondence should be addressed: Department of Cancer Biology, Instituto de Investigaciones Biomédicas, Consejo Superior de Investigaciones Científicas and Universidad Autónoma de Madrid, c/ Arturo Duperier 4, E-28029 Madrid, Spain. Phone: +34-91-585-4424; Fax: +34-91585-4401; E-mail: antonio.villalobo@iib.uam.es
\end{abstract}

Running title: EGFR O-GIcNAcylation

Key words: Antibodies, click-chemistry, epidermal growth factor receptor, $N$ glycans, O-GlcNAcylation, tunicamycin. 


\section{Abstract}

The reversible $O$-linked attachment of single $\beta$-D-N-acetylglucosamine (GIcNAc) moieties to serine/threonine residues in target proteins is a frequently occurring post-translational modification affecting the functionality of many cellular systems. In this report we present experimental evidences suggesting that the epidermal growth factor receptor (EGFR) is subjected to O-GlcNAcylation in human carcinoma epidermoid A431 and human lung carcinoma A549 cells. However, no signal was detected in human cervix adenocarcinoma HeLa cells or in mouse EGFR-T17 fibroblasts ectopically expressing the human EGFR. We detected a positive O-GIcNAcylation signal in immunoprecipitated EGFR by Western blot using two distinct specific anti-O-GIcNAc antibodies even after $N$ deglycosylation of the receptor using peptide- $N$-glycosidase $F$ (PNGase F). Conversely, the presence of EGFR was detected by Western blot using an antiEGFR antibody in the immunocomplex of O-GlcNAcylated proteins immunoprecipitated with an anti-O-GIcNAc antibody. These signals were enhanced when the O-linked $\beta-N$-acetylglucosaminidase (OGA) inhibitor Thiamet $G$ was added to prevent the deglycosylation of the GlcNAc moiety(ies). Moreover, we also detected a positive signal in the immunoprecipitated and $\mathrm{N}$ deglycosylated EGFR using PNGase F, and tunicamycin when the cells were metabolically labeled with azido-GIcNAc (GIcNAz), biotinylated and probed with streptavidin-labeled peroxidase. Finally, EGFR and OGT co-immunoprecipitate, and incubation of immunoprecipitated EGFR with immunoprecipitated O-linked $\beta-N$-acetylglucosamine transferase (OGT) in the presence of uridine 5 'diphospho- $N$-acetylglucosamine (UDP-GlcNAc) resulted in a significant enhancement of the EGFR O-GlcNAcylation signal as detected by Western blot using an anti-O-GIcNAc antibody. We conclude that the human EGFR is subjected to O-GlcNAcylation in the A431 and A549 tumor cell lines.

\section{Introduction}

Protein O-GlcNAcylation is a post-translational modification first identified in lymphocyte proteins ${ }^{1}$, consisting in the reversible $O$-linked attachment of 
single $\beta$-D- $N$-acetylglucosamine (GICNAc) moieties to serine/threonine residues in target proteins; thus modifying their enzymatic activity, intracellular location, turnover, and/or other functional roles $^{2}$. Since those early days, literally hundreds of additional cytosolic, nuclear and mitochondrial proteins have been identified to be O-GlcNAcylated, and it is likely that this number would be growing in the future. Altered O-GIcNAcylation has been proposed to contribute to the etiology of many illnesses such type-2 diabetes ${ }^{3-6}$, cardiovascular ailments $^{3,7}$, neurodegeneration as in Alzheimer's disease ${ }^{3,4,8,9}$ and cancer ${ }^{4,10-}$ 13.

The enzymes $O$-linked $\beta-N$-acetylglucosamine transferase $(\mathrm{OGT})^{14}$ and O-linked $\beta-N$-acetylglucosaminidase $(O G A)^{15}$ catalyze the reversible $O$ GlcNAcylation of proteins, the first one doing the glycosylation and the latter one the deglycosylation. Several OGT isoforms are present in the cell, more relevantly a canonical long form localized in the nucleus and cytosol (ncOGT), another one a bit shorter localized in the inner mitochondrial membrane (mOGT), and a third one, the shortest of them (sOGT), also localized in the nucleus and cytosol ${ }^{14}$. In addition, an atypical epidermal growth factor (EGF)like-repeat domain specific OGT (EOGT) able to O-GIcNAcylate the side chain of Ser/Thr at the extracellular segment of different proteins, including Dumpy in Drosophila and the Notch receptor in mouse, has been identified ${ }^{16-18}$.

Protein O-GlcNAcylation appears to be at the same footing than protein phosphorylation in terms of importance in regulating signal transduction events; and indeed it exists a complex crosstalk between both events taking place either at the same site, or at distinct adjacent, proximal or distant sites, competing or facilitating each other in the choreographic orchestration of signaling fluxes regulating multiple pathways implicated in the physiology of the cell, such as: metabolic control, stress response, epigenetic modification, gene transcription, translation of proteins, protein turnover, differentiation, apoptosis and cell cycle control to mention a few ${ }^{4}{ }^{19-22}$. Moreover, O-GIcNAcylation of some proteins can also affect its phosphorylation at tyrosine residues, as it is the case of prohibitin ${ }^{23}$. 
Among the OGT substrates a large set of serine/threonine- and tyrosinekinases were identified, amounting to forty-two of a total of 152 candidate kinases tested in a particular study ${ }^{24}$. Moreover, and of interest to the present study, the insulin receptor, a relevant member of the receptor tyrosine kinase (RTK) superfamily, is also subjected to O-GlcNAcylation, process that modulates the functionality of the receptor and down-stream signaling pathways $^{25}$. However, little information is available on O-GlcNAcylation of other RTKs.

The epidermal growth factor receptor (EGFR) is an RTK implicated in the control of cell proliferation, differentiation, cell survival, programmed cell death and cell migration processes, and this receptor is frequently overexpressed or mutated in many solid tumors thus contributing to cancerogenesis ${ }^{26,27}$. In an early report, using high throughput proteomic analysis, labeling proteins of Drosophila melanogaster with the GlcNAc analogue azido-GlcNAc, the EGFR type III was identified, suggesting that it was susceptible to undergo $O$ GlcNAcylation ${ }^{28}$. Thereafter, in an entirely in silico study it was proposed that the human EGFR could be subjected to O-GlcNAcylation at Thr654 and Ser1046/Ser1047 $7^{29}$, phosphorylation sites of protein kinase C (PKC) ${ }^{30}$ and calmodulin-dependent protein kinase II (CaMK-II) ${ }^{31}$, respectively. However, experimental evidence for this proposed post-translational modification of human EGFR is lacking up to day. In this report we shall provide for the first time proof that indeed the human EGFR could be subjected to O-GIcNAcylation in two distinct tumor cell lines, discuss the uncertainties pending to be resolved on the occurrence of this post-translational modification in the EGFR, and suggest potential physiological consequence of this event for the functionality of the receptor.

\section{Material and Methods}

Reagents and antibodies

Benzyl 2-acetamido-2-deoxy- $\alpha$-D-galactopyranoside (BADGP), O-(2acetamido-2-deoxy-D-glucopyranosylidene)amino $N$-phenyl carbamate (PUGNAc), 3aR,5R,6S,7R,7aR)-2-(ethylamino)-3a, 6,7,7a-tetrahydro-5- 
(hydroxymethyl)-5H-pyrano[3,2-d]thiazole-6,7-diol (Thiamet G), tunicamycin, UDP-GlcNAc (sodium salt), anti-mouse IgG (FC specific) horseradish peroxidase (HRP)-conjugated secondary antibody, mouse monoclonal antitubulin- $\alpha$ (DM1A) antibody and purified mouse IgG (technical grade) were obtained from Sigma-Aldrich Co. (St. Louis, MO). Mouse monoclonal antiphosphotyrosine antibody (4G10, isotype $\operatorname{lgG}_{2 b \kappa}$ ) and rabbit monoclonal antiEGFR (E235) antibody (recognizing the C-terminal domain of EGFR) were obtained from Millipore (Billerica, MA). Rabbit monoclonal anti-glyceraldehyde 3-phosphate dehydrogenase (GAPDH) (14C10) antibody was obtained from Cell Signalling. Anti-rabbit IgG HRP-linked secondary antibody, streptavidin peroxidase-conjugated tetraacetylated $N$-azidoacetylglucosamine (GlcNAz) and Click-iT®-protein reaction buffer kit were obtained from Invitrogen (Eugene, OR). The classic magnetic IP/Co-IP kit, the O-GIcNAc Western blot detection kit containing the mouse monoclonal anti-O-GIcNAc monoclonal antibody (CTD110.6) and goat anti-mouse $\lg \mathrm{M}$ ( $\mu$ heavy chain) HRP-conjugated secondary antibody were obtained from Thermo Scientific-Pierce (Rockford, IL). The O-GIcNAc antibody (RL2) was obtained from Novus Biologicals (Littleton, USA). Anti-OGT antibody (ab50271) was obtained from Abcam (Cambridge, UK) and peptide-N-glycosidase F (PNGase F) (from Flavobacterium meningosepticum) was obtained from New England BioLabs (Herts, UK).

\section{Cell culture}

Human carcinoma epidermoid A431 cells, human lung carcinoma A549 cells, human cervix adenocarcinoma HeLa cells, and mouse EGFR-T17 fibroblast stably transfected with the human EGFR were routinely cultured in Dulbecco's modified Eagle's medium (DMEM) supplemented with $10 \%$ (v/v) fetal bovine serum (FBS), $2 \mathrm{mM}$ L-glutamine and $40 \mu \mathrm{g} / \mathrm{ml}$ gentamicin at $37^{\circ} \mathrm{C}$ in a humidified air atmosphere containing $5 \% \mathrm{CO}_{2}$.

\section{O-GICNAc detection in EGFR}

EGFR was immunoprecipitated from the different cell lines using the Pierce Classic Magnetic IP/Co-IP kit following the manufacturer instructions. Briefly, the cell lysates (1-2 mg proteins) were incubated with anti-EGFR antibody (1:500 dilution) overnight at $4^{\circ} \mathrm{C}$. The lysate containing the antigen- 
antibody complex was mixed with the magnetic beads and incubated for 1 hour at room temperature. The beads were extensively washed and the EGFR was released with the low $\mathrm{pH}(\mathrm{pH}-2)$ elution buffer, resolved in polyacrylamide gel electrophoresis in the presence of sodium dodecyl sulfate (SDS-PAGE) and subjected to Western blot using appropriate anti-O-GlcNAc antibodies (RL2 or CTD110.6). Conversely, a lysate of A431 cells (1-2 mg proteins) was incubated as above with an anti-O-GIcNAc antibody (RL2) and the immunoprecipitated $O-$ GIcNAcylated proteins were resolved in SDS-PAGE and subjected to Western blot using and anti-EGFR antibody.

\section{EGFR and OGT co-immunoprecipitation}

Co-immunoprecipitation of EGFR and OGT from A431 cells (2 mg protein) was performed using the Pierce ${ }^{\mathrm{TM}}$ Classic Magnetic IP/Co-IP kit and either anti-EGFR or anti-OGT antibodies following the manufacture instructions. The samples were processed for SDS-PAGE and Western blot using both antiEGFR and anti-OGT antibodies to detect co-immunoprecipitation of the two proteins.

\section{In vitro EGFR O-GIcNAcylation}

Immunoprecipitated EGFR was used as substrate for in vitro $O$ GlcNAcylation assay using immunoprecipitated OGT from A431 cells. The OGlcNAcylation reaction took place in a buffer containing $50 \mathrm{mM}$ tris(hydroxymethyl)aminomethane (Tris)- $\mathrm{HCl}(\mathrm{pH} 7.5), 1 \mathrm{mM}$ dithiotreitol, 12.5 $\mathrm{mM} \mathrm{MgCl} 2$ and $1 \mathrm{mM}$ UDP-GICNAc, and incubated for $2.5 \mathrm{~h}$ at $30{ }^{\circ} \mathrm{C}$. The reaction was stopped by adding Laemmli buffer and boiled for $10 \mathrm{~min}$ at $100{ }^{\circ} \mathrm{C}$, resolved in SDS-PAGE, transferred to a polyvinylidene difluoride (PVDF) membrane, and probed with an anti-O-GIcNAc antibody (RL2).

\section{Metabolic protein labeling with GIcNAz}

A431 cells were seeded in $150 \mathrm{~cm}^{2}$ petri dishes and when $\sim 70 \%$ confluence was attained $40 \mu \mathrm{M}$ GlcNAz was added and incubated for $72 \mathrm{~h}$. The cells were lysed in a buffer containing $25 \mathrm{mM}$ Tris- $\mathrm{HCl}(\mathrm{pH} 7.4), 150 \mathrm{mM} \mathrm{NaCl}$, $1 \mathrm{mM}$ ethylenediaminetetraacetic acid (EDTA), $1 \%$ (v/v) nonyl phenoxypolyethoxylethanol (NP-40) and $5 \%(\mathrm{v} / \mathrm{v})$ glycerol supplemented with 
$20 \mu \mathrm{M}$ PUGNAc to prevent deglycosylation and a cocktail of protease inhibitors:

$0.5 \mathrm{mM} 4$-(2-aminoethyl) benzenesulfonyl fluoride (AEBSF), $0.4 \mu \mathrm{M}$ aprotinin, $25 \mu \mathrm{M}$ bestatin, $7.5 \mu \mathrm{M} \quad$ [1-[N-[(L-3-trans-carboxyoxirane-2-carbonyl)-Lleucyl]amino]-4-guanidinobutane] (E-64), $10 \mu \mathrm{M}$ leupeptin, $5 \mu \mathrm{M}$ pepstatin A, and freshly prepared $0.6 \mathrm{mM}$ phenylmethylsulfonyl fluoride (PMSF). The lysate was centrifuged at $16,000 \mathrm{~g}$ for $10 \mathrm{~min}$ and the supernatant used for EGFR immunoprecipitation. Biotinylation of the GlcNAz-labeled proteins was performed using the Click-iT®-Protein Reaction Buffer kit following the manufacturer's instructions, the samples were subjected to SDS-PAGE, the proteins transferred to PVDF and overlaid with streptavidin-HRP $(1: 10,000$ dilution).

\section{$N$-deglycosylation of the EGFR}

The immunoprecipitated EGFR was first denatured in a buffer containing $0.5 \%$ (w/v) SDS and $40 \mathrm{mM}$ dithiothreitol and boiled for $10 \mathrm{~min}$ at $100{ }^{\circ} \mathrm{C}$. Thereafter, $10 \%(\mathrm{v} / \mathrm{v})$ NP-40, $50 \mathrm{mM}$ sodium phosphate $(\mathrm{pH} \mathrm{7.5)}$ and 1,000 units of PNGase F were added, and the mixture was incubated for $2 \mathrm{~h}$ at $37^{\circ} \mathrm{C}$. One unit of PNGase $F$ is defined as the amount of enzyme required to remove $>95 \%$ of the carbohydrates from $10 \mu \mathrm{g}$ of denatured RNase B in 1 hour at 37 ${ }^{\circ} \mathrm{C}$. The reaction was stopped by the addition of Laemmli buffer and boiled for $10 \mathrm{~min}$ at $100{ }^{\circ} \mathrm{C}$, resolved in SDS-PAGE, transferred to a PVDF membrane and probed with anti-O-GICNAc antibodies (RL2 or CTD110.6). When tunicamycin was used as a deglycosylation agent A431 cells were cultured in complete DMEM supplemented with $1 \mu \mathrm{g} / \mathrm{ml}$ tunicamycin overnight where only newly synthetized EGFR molecules are expected to be fully $N$-deglycosylated. Higher concentrations of tunicamycin to attain more $N$-deglycosylated EGFR molecules was not possible as more drastic treatments result in extensive cell death.

\section{Effect of OGT and OGA inhibitors on EGFR O-GIcNAcylation in living cells}

A431 cells were grown to confluence in 6-well culture dishes containing 2 $\mathrm{ml}$ of DMEM supplemented with $10 \%(\mathrm{v} / \mathrm{v})$ FBS overnight in the absence and presence of the OGT inhibitor BADGP (2 mM), or the OGA inhibitor Thiamet G $(20 \mu \mathrm{M})$. Thereafter, the EGFR was immunoprecipitated from a cell lysate using 
an anti-EGFR antibody and the immunocomplex was processed by Western blot using an anti-O-GlcNAc antibody (RL2). Alternatively, O-GlcNAcylated proteins were immunoprecipitated using an anti-O-GlcNAc antibody (RL2) from a cell lysate and the immunocomplex was processed by Western blot using an anti-EGFR antibody. The effect of $2 \mathrm{mM}$ BADGP and increasing concentrations (0.5-20 $\mu \mathrm{M})$ of Thiamet $\mathrm{G}$ was also tested on total proteins O-GlcNAcylation level from an A431 cell lysate as positive control.

Polyacrylamide gel electrophoresis and Western blot

Proteins were separated by SDS-PAGE in a 5-20\% (w/v) linear gradient slab gel of polyacrylamide and $0.1(\mathrm{w} / \mathrm{v})$ sodium dodecyl sulfate $(\mathrm{pH} 8.3)$ at 6-10 $\mathrm{mA}$ overnight ${ }^{32}$. The proteins were electrotransferred from the gels to a PVDF membrane for $2 \mathrm{~h}$ at $300 \mathrm{~mA}$ in a medium containing $48 \mathrm{mM}$ Tris-base, 36.6 $\mathrm{mM}$ L-glycine, $0.04 \%(\mathrm{w} / \mathrm{v})$ SDS and $20 \%(\mathrm{v} / \mathrm{v})$ methanol. The proteins were fixed with $0.2 \%(\mathrm{v} / \mathrm{v})$ glutaraldehyde in $25 \mathrm{mM}$ Tris- $\mathrm{HCl}(\mathrm{pH} 8), 150 \mathrm{mM} \mathrm{NaCl}$ and $2.7 \mathrm{mM} \mathrm{KCl}$ (TNK buffer) for $45 \mathrm{~min}$, and transiently stained with Fast Green to ascertain the regularity of the transfer procedure. The PVDF membranes were blocked with $5 \%$ (w/v) bovine serum albumin or $5 \%(\mathrm{w} / \mathrm{v})$ fatfree powdered milk following the instructions of the antibodies' manufacturers in $0.1 \%$ (w/v) Tween-20, $100 \mathrm{mM}$ Tris- $\mathrm{HCl}(\mathrm{pH} 8.8), 500 \mathrm{mM} \mathrm{NaCl}$ and $0.25 \mathrm{mM}$ $\mathrm{KCl}$ (T-TBS buffer), and probed overnight at $4^{\circ} \mathrm{C}$ using a $1 / 2000$ dilution of the corresponding primary antibody, and for $1 \mathrm{~h}$ at room temperature using a $1 / 5000$ dilution of the appropriate secondary anti-IgG or anti-IgM antibodies coupled to HRP. The bands were visualized upon development with an enhanced chemiluminescence kit, following instructions from the manufacturer, and exposure of x-ray films for appropriate periods of time. The intensity of the bands was quantified using the ImageJ 1.46r program (National Institutes of Health, USA).

\section{Statistical analysis}

The two-tailed paired Student's t test was performed using the Microsoft Excel (Microsoft Co., Redmon, WA) software program. Data were expressed as the mean \pm SEM and differences were considered significant at $p \leq 0.05$. 


\section{Results}

Protein O-GlcNAcylation in A431 tumor cells

We first tested for the presence of O-GlcNAcylated proteins in whole cell lysates of A431 tumor cells by Western using two distinct (CTD110.6 and RL2) anti-O-GIcNAc specific antibodies (Fig. 1A). The presence of multiple strongly labeled bands of O-GlcNAcylated proteins in a range of $>250 \mathrm{kDa}$ to $<50 \mathrm{kDa}$ were detected presenting a similar distribution pattern when both antibodies were used. Fainter bands were also detected in overexposed films (not shown). When cells were treated with Thiamet G, a highly specific OGA inhibitor to prevent $O-G I c N A c$ deglycosylation ${ }^{33}$, a significant two-fold increase in protein O-GlcNAcylation level was detected as expected (Figs. 1B-1D). Moreover, we also tested BADGP, a general O-glycosylation inhibitor not specific for $O$ GlcNAcylation but that also inhibits this process ${ }^{34-37}$, and a small but significant decrease in protein O-GICNAcylation was detected (Figs. 1B, 1C).

\section{EGFR O-GIcNAcylation detected by immunoblots}

To determine whether the EGFR from A431 tumor cells undergoes $O$ GlcNAcylation we treated the immunoprecipitated receptor in the absence and presence of PNGase $\mathrm{F}$ to remove $\mathrm{N}$-glycans, and tested for O-GlcNAcylation using the same two distinct anti-O-GlcNAc antibodies mentioned above (Figs. $2 \mathrm{~A}, 2 \mathrm{~B})$. The results show that in both instances, not only the $170 \mathrm{kDa}$ native EGFR, but most significantly and in greater extent the $\sim 150 \mathrm{kDa} N$ deglycosylated receptor, yielded positive signals. To ascertain the specificity of the CTD110.6 anti-O-GICNAc antibody, we competed its reactivity using an excess (20 mM) of free GlcNAc in the incubation medium (Fig. 2C).

Furthermore, we performed the experiment in reverse, immunoprecipitating first O-GlcNAcylated proteins from an A431 cell lysate and detecting the presence of EGFR in the immunoprecipitate (Fig. 2D). When the cells were previously treated with the OGA inhibitor Thiamet G, the EGFR signal was slightly increased, although not significant decrease was detected when cells were treated with the O-glycosylation inhibitor BADGP (Fig. 2D). In agreement with this, we also demonstrated that when A431 cells were treated 
with Thiamet G the O-GIcNAcylation level of the immunoprecipitated native 170 kDa EGFR also increased, although little if any increment was detected in the 150 kDa N-glycans-free EGFR band (Figs. 3A, 3B).

\section{EGFR O-GIcNAcylation detected by metabolic labeling with azido-GIcNAc}

We next tried to detect O-GIcNAcylation of the EGFR by metabolically labeling cellular glycans in proteins from A431 cells with azido-GIcNAc. Fig. 4A shows that after immunoprecipitation both the native $170 \mathrm{kDa}$ EGFR and its $\sim 150$ kDa N-glycans-free form, obtained after PNGase F treatment, yielded a positive signal following biotinylation and overlay by streptavidin-HRP. This was observed in basal conditions and after EGF addition. The extent of the signal was lower in EGF-treated cells but this most likely represents degradation of the receptor after ligand-induced internalization, as this process has been amply documented 38,39 , and we further document this degradation in Figs. 4B, 4C. We also treated A431 cells with tunicamycin to obtain EGFR devoid of $N$ glycans by a different method. Fig. 4D shows that the native $170 \mathrm{kDa}$ receptor and its $\sim 150 \mathrm{kDa} N$-deglycosylated form both were metabolically labeled with azido-GlcNAc, even after further treating the immunoprecipitate EGFR with PNGase F.

\section{EGFR O-GlcNAcylation in different cell lines}

We tested different human tumor cell lines, with distinct levels of EGFR expression, and a mouse fibroblast stably transfected and overexpressing the human EGFR for O-GICNAcylation of the receptor. Fig. 5 shows a positive signal in human carcinoma epidermoid A431 cells, which were used as positive control. Also, a positive signal was detected in human lung carcinoma A549 cells. The double band observed in A549 cells may represent the presence of an unrelated O-GIcNAcylated protein associated to the EGFR and/or another ErbB receptor forming heterodimers with the EGFR. However, no OGlcNAcylation of the EGFR was detected in human cervix adenocarcinoma HeLa cells or in the recombinant human EGFR ectopically expressed in mouse EGFR-T17 fibroblasts. 
To attain a further proof that the EGFR could be O-GlcNAcylated, we assayed in vitro the enzymatic activity of immunoprecipitated OGT from A431 cells using as substrate the immunoprecipitated EGFR from the same origin. We first show that EGFR and OGT can be co-immunoprecipitated (Fig. 6A), suggesting that the two proteins interact in living cells. Our results on the in vitro enzymatic assays show a significant increment in the O-GlcNAcylation level of the EGFR as detected by Western blot using an anti-O-GIcNAc antibody (RL2) (Figs. 6B, 6C). Given the fact that the immunoprecipitated EGFR was already O-GIcNAcylated before starting the reaction catalyzed by OGT, this increment only reached $\sim 1.8 \pm 0.2$ fold. This, nevertheless, strongly suggests that the EGFR undergoes this post-translational modification.

\section{Discussion}

Overall our results show for the first time that human EGFR could be subjected to O-GIcNAcylation, as it is the case for the insulin receptor ${ }^{25}$. We detected EGFR O-GlcNacylation in two distinct human tumor cell lines, A431 and A549 cells. The first one, derived from a tumor of epidermic origin, and the second one from a lung epithelial tumor. In contrast, no signal was detected in HeLa cells that are derived from a cervical adenocarcinoma, and therefore are from glandular origin. This demonstrates that EGFR O-GIcNacylation may not occur in all tumor cells as this process could depend of its tissular and/or organ origin. Moreover, the absence EGFR O-GlcNacylation signal in EGFR-T17 fibroblasts suggests that this post-translational modification may not be operative in a non-physiological setting where a recombinant human EGFR is artificially expressed in cells from another species.

This post-translational modification is expected to take place in the cytosolic region of the EGFR. Nevertheless, it is noticeable that when the immunoprecipitated EGFR was treated with PNGase $F$ the immunoreactivity of the $\sim 150 \mathrm{kDa} N$-deglycosylated receptor to the two anti-O-GlcNAc antibodies used increased as compared with the native $170 \mathrm{kDa}$ EGFR. This suggests that removal of $N$-glycans from the extracellular region of the EGFR could facilitate the exposure of occluded GIcNAc moieties linked to complex O-glycans, not 
related to O-GIcNAcylation events but that could be non-specifically recognized by the two anti-O-GIcNAc antibodies used in this study. In fact, the presence of O-glycans in the extracellular region of the EGFR has been described ${ }^{40}$. Alternatively, the increased immunoreactivity of the anti-O-GIcNAc antibodies after the removal of the $N$-glycans by PNGase F, could be also related to the extracellular O-GIcNAcylation of the receptor, modification driven by EOGT ${ }^{16-18}$. We identified in the extracellular region of the EGFR three sites $\left({ }^{215}\right.$ CAQQCSGRC ${ }^{223},{ }^{236}$ CAAGCTGPRE $^{245}$ and $\left.{ }^{506} \mathbf{C H A L C S P E G C}^{515}\right)$ that contain key amino acids (cysteine, glycine and serine/threonine) that are also present in the EGF-like domain consensus sequence CXXGXS/TGXXC recognized by $\mathrm{EOGT}^{41}$. These sites are in the $\mathrm{S} 1$ and $\mathrm{S} 2$ cysteine-rich regions of the receptor, and the cysteine residues located in the proposed sequences are involved in the formation of disulfide bridges $^{42}$. Moreover, the strict specificity of some of the anti-O-GIcNAc antibodies has been questioned, as for example the CTD110.6 antibody that also recognizes terminal $\beta$-GICNac in complex $N$-glycans ${ }^{43}$.

PNGase $\mathrm{F}$ is extensively used to remove $N$-glycans from glycoproteins ${ }^{44}$, but this endoglycosidase is unable to work if a $\alpha 1-3$ bond links fucose to the innermost GlcNAc attached to asparagine ${ }^{45}$. Although $\alpha 1-3$-linked fucose to the innermost GICNAc residues attached to asparagine is common in plant and insect glycoproteins, it appears to be absent in mammalian cells, where $\alpha 1-6$ linked fucosylation of core GlcNAc catalyzed by fucosyl transferase 8 (FUT8) appears to be the norm ${ }^{46}$. Nevertheless, to eliminate the possible existence of an unrecognized aberrant $\alpha 1-3-$ linked fucosylation of the innermost GIcNAc attached to asparagine in tumor cells we used tunicamycin treatment. Our results with tunicamycin also supports absence of $\alpha 1$-3-linked fucosylation of core GIcNAc in the EGFR from A431 cells, as GlcNAz labeling was clearly detected in the $\sim 150 \mathrm{kDa} N$-deglycosylated EGFR.

The suggestion that the EGFR could be subjected to O-GlcNAcylation at Thr654 and Ser1046/Ser1047 based on in silico modeling as proposed by Kaleem et al. $^{29}$ is pending experimental confirmation, as many predicting 
software programs are prone to both false-positive and false-negative predictions ${ }^{47}$. We attempted some experiments using mass spectrometry (MS) to detect EGFR O-GlcNAcylation. However, the lack of detected peptides corresponding to the cytosolic juxtamembrane region of the EGFR, where Thr654 is located, prevented attaining meaningful results. We believe that the identification in human EGFR of O-linked GICNAc moiety/ies and the specific Ser and/or Thr residue(s) where it may be attached using a suitable MS method or another technology could be, if positive, the ultimate proof of EGFR OGlcNAcylation.

If Thr654 were to be confirmed as an O-GIcNAcylation site in EGFR, an interesting subject would be to elucidate the possible tripartite crosstalk that may be established between the phosphorylation of this site by $\mathrm{PKC}^{30}$, the binding of calmodulin ( $\mathrm{CaM}$ ) to the cytosolic juxtamembrane region of the receptor where the Thr654 residue is located ${ }^{48}$, and the O-GIcNAcylation event. We have demonstrated that phosphorylation of Thr654 by PKC prevents CaM binding to this region, and conversely binding of CaM prevents Thr654 phosphorylation by $\mathrm{PKC}^{48}$. Thus, it is expected that if Thr654 were targeted by OGT a more complex functional picture could emerge by the presence of this O-GlcNAcylated residue. Particularly, since phosphorylation of Thr654 appears to inhibit ligand-dependent EGFR activation and maintaining the receptor in stand-by at the plasma membrane slowing its internalization ${ }^{49}$, and $\mathrm{CaM}$ binding appears to contribute to ligand-dependent EGFR activation in living cells ${ }^{50}$.

Finally, another important issue to be studied in future work would be to establish the potential impact that O-GIcNAcylation may have on EGFR functionality, particularly its putative action on ligand-dependent activation, receptor downregulation, membrane location, nuclear translocation, internalization, intracellular traffic and/or degradation via the lysosomal and/or the ubiquitin-proteasome pathways. Interestingly, it is known that the EGFR can be located in non-caveloar lipid rafts ${ }^{51}$, as also occurs with a fraction of the $O$ GlcNAc transferase when cells are stimulated with insulin ${ }^{52}$. This suggests that the co-localization of OGT and the EGFR at the same membrane microdomains could facilitate the O-GICNAcylation of the receptor. How these events 
may affect important EGFR-mediated cellular functions, such as cell proliferation, differentiation, cell survival, programmed cell death and/or cell migration processes warrant also special attention, as well as how this posttranslational modification of the EGFR may goes awry in pathological conditions such as cancer.

\section{Conclusion}

We conclude that the accumulated experimental evidences presented in this report using different techniques strongly suggest that the human EGFR from A431 and A549 tumor cells is subjected to O-GlcNAcylation. Nevertheless, the identification of the serine and/or threonine residue(s) implicated in this post-translational modification, and the role that this process may have in the functionality of the EGFR and the physiology of the cell, particularly EGFRmediated cell proliferation, survival, differentiation, and/or cell motility, are important issues to be study in future work. Moreover, since altered signaling processes is known to occur in tumor cells, it would also be of great interest to determine whether this particular O-GIcNAcylation detected in the EGFR is an aberrant process happening only in some cancerous cells, or by contrast is a physiological process also occurring in the receptor of non-transformed cells.

\section{Acknowledgements}

This work was funded by grants to AV from the European Commission (contract No. PITN-GA-2011-289033), the Secretaría de Estado de Investigación, Desarrollo e Innovación (SAF2011-23494 and SAF2014-52048-R), and the Consejería de Educación de la Comunidad de Madrid (S2011/BMD-2349). SRS received funding from the People Program (Marie Curie Actions) of the European Union's Seventh Framework Program FP7/2007-2013 under REA grant agreement PITN-GA-2011-289033.

\section{Bibliographic references}

1. C. R. Torres and G. W. Hart, J Biol Chem, 1984, 259, 3308-3317.

2. G. W. Hart, J Biol Chem, 2014, 289, 34422-34423. 
3. T. Lefebvre, V. Dehennaut, C. Guinez, S. Olivier, L. Drougat, A. M. Mir, M. Mortuaire, A. S. Vercoutter-Edouart and J. C. Michalski, Biochim Biophys Acta, 2010, 1800, 67-79.

4. G. W. Hart, C. Slawson, G. Ramirez-Correa and O. Lagerlof, Annu Rev Biochem, 2011, 80, 825-858.

5. J. Ma and G. W. Hart, Expert Rev Proteomics, 2013, 10, 365-380.

6. K. Vaidyanathan and L. Wells, J Biol Chem, 2014, 289, 34466-34471.

7. S. A. Marsh, H. E. Collins and J. C. Chatham, J Biol Chem, 2014, 289, 34449-34456.

8. C. X. Gong, F. Liu, I. Grundke-Iqbal and K. Iqbal, J Alzheimers Dis, 2006, 9, 1-12.

9. Y. Zhu, X. Shan, S. A. Yuzwa and D. J. Vocadlo, J Biol Chem, 2014, 289, 34472-34481.

10. Z. Li and W. Yi, Glycoconj J, 2014, 31, 185-191.

11. P. Jozwiak, E. Forma, M. Brys and A. Krzeslak, Front Endocrinol (Lausanne), 2014, 5, 145.

12. R. M. de Queiroz, E. Carvalho and W. B. Dias, Front Oncol, 2014, 4, 132.

13. Z. Ma and K. Vosseller, J Biol Chem, 2014, 289, 34457-34465.

14. J. Janetzko and S. Walker, J Biol Chem, 2014, 289, 34424-34432.

15. J. Alonso, M. Schimpl and D. M. van Aalten, J Biol Chem, 2014, 289, 34433-34439.

16. Y. Sakaidani, T. Nomura, A. Matsuura, M. Ito, E. Suzuki, K. Murakami, D. Nadano, T. Matsuda, K. Furukawa and T. Okajima, Nat Commun, 2011, 2, 583.

17. Y. Sakaidani, N. Ichiyanagi, C. Saito, T. Nomura, M. Ito, Y. Nishio, D. Nadano, T. Matsuda, K. Furukawa and T. Okajima, Biochem Biophys Res Commun, 2012, 419, 14-19.

18. M. Ogawa, K. Furukawa and T. Okajima, World J Biol Chem, 2014, 5, 224230.

19. Z. Wang, M. Gucek and G. W. Hart, Proc Natl Acad Sci U S A, 2008, 105, 13793-13798.

20. Q. Zeidan and G. W. Hart, J Cell Sci, 2010, 123, 13-22.

21. G. W. Hart, C. Slawson, G. Ramirez-Correa and O. Lagerlof, Annu Rev Biochem, 2011, 80, 825-858.

22. B. A. Lewis and J. A. Hanover, J Biol Chem, 2014, 289, 34440-34448.

23. S. R. Ande, S. Moulik and S. Mishra, PLoS One, 2009, 4, e4586.

24. W. B. Dias, W. D. Cheung and G. W. Hart, Biochem Biophys Res Commun, 2012, 422, 224-228.

25. X. Yang, P. P. Ongusaha, P. D. Miles, J. C. Havstad, F. Zhang, W. V. So, J. E. Kudlow, R. H. Michell, J. M. Olefsky, S. J. Field and R. M. Evans, Nature, 2008, 451, 964-969.

26. R. N. Jorissen, F. Walker, N. Pouliot, T. P. Garrett, C. W. Ward and A. W. Burgess, Exp Cell Res, 2003, 284, 31-53.

27. M. A. Lemmon, J. Schlessinger and K. M. Ferguson, Cold Spring Harb Perspect Biol, 2014, 6, a020768.

28. R. Sprung, A. Nandi, Y. Chen, S. C. Kim, D. Barma, J. R. Falck and Y. Zhao, J Proteome Res, 2005, 4, 950-957.

29. A. Kaleem, I. Ahmad, D. C. Hoessli, E. Walker-Nasir, M. Saleem, A. R. Shakoori and D. Nasir Ud, Mol Biol Rep, 2009, 36, 631-639.

30. T. Hunter, N. Ling and J. A. Cooper, Nature, 1984, 311, 480-483. 
31. J. L. Countaway, A. C. Nairn and R. J. Davis, J Biol Chem, 1992, 267, 11291140.

32. U. K. Laemmli, Nature, 1970, 227, 680-685.

33. H. Goldberg, C. Whiteside and I. G. Fantus, Am J Physiol Endocrinol Metab, 2011, 301, E713-726.

34. G. Filhoulaud, G. Guillemain and R. Scharfmann, J Biol Chem, 2009, 284, 24583-24594.

35. M. Pantaleon, H. Y. Tan, G. R. Kafer and P. L. Kaye, Biol Reprod, 2010, 82, 751-758.

36. Y. Onodera, J. M. Nam and M. J. Bissell, J Clin Invest, 2014, 124, 367-384.

37. Q. Xu, C. Yang, Y. Du, Y. Chen, H. Liu, M. Deng, H. Zhang, L. Zhang, T. Liu, Q. Liu, L. Wang, Z. Lou and H. Pei, Nucleic Acids Res, 2014, 42, 55945604.

38. A. M. Soderquist and G. Carpenter, J Membr Biol, 1986, 90, 97-105.

39. A. Kirisits, D. Pils and M. Krainer, Int J Biochem Cell Biol, 2007, 39, 21732182.

40. Y. M. Wu, C. H. Liu, R. H. Hu, M. J. Huang, J. J. Lee, C. H. Chen, J. Huang, H. S. Lai, P. H. Lee, W. M. Hsu, H. C. Huang and M. C. Huang, Cancer Res, 2011, 71, 7270-7279.

41. J. F. Alfaro, C. X. Gong, M. E. Monroe, J. T. Aldrich, T. R. Clauss, S. O. Purvine, Z. Wang, D. G. Camp, 2nd, J. Shabanowitz, P. Stanley, G. W. Hart, D. F. Hunt, F. Yang and R. D. Smith, Proc Natl Acad Sci U S A, 2012, 109, 7280-7285.

42. Y. Abe, M. Odaka, F. Inagaki, I. Lax, J. Schlessinger and D. Kohda, J Biol Chem, 1998, 273, 11150-11157.

43. Y. Tashima and P. Stanley, J Biol Chem, 2014, 289, 11132-11142.

44. F. Maley, R. B. Trimble, A. L. Tarentino and T. H. Plummer, Jr., Anal Biochem, 1989, 180, 195-204.

45. V. Tretter, F. Altmann and L. Marz, Eur J Biochem, 1991, 199, 647-652.

46. B. Ma, J. L. Simala-Grant and D. E. Taylor, Glycobiology, 2006, 16, 158R184R.

47. R. Jochmann, P. Holz, H. Sticht and M. Sturzl, Biochim Biophys Acta, 2014, 1844, 416-421.

48. J. Martin-Nieto and A. Villalobo, Biochemistry, 1998, 37, 227-236.

49. P. Sánchez-González, K. Jellali and A. Villalobo, FEBS J, 2010, 277, $327-$ 342.

50. H. Li, S. Panina, A. Kaur, M. J. Ruano, P. Sanchez-Gonzalez, J. M. la Cour, A. Stephan, U. H. Olesen, M. W. Berchtold and A. Villalobo, J Biol Chem, 2012, 287, 3273-3281.

51. K. Roepstorff, P. Thomsen, K. Sandvig and B. van Deurs, J Biol Chem, 2002, 277, 18954-18960.

52. Y. Perez-Cervera, V. Dehennaut, M. Aquino Gil, K. Guedri, C. J. Solorzano Mata, S. Olivier-Van Stichelen, J. C. Michalski, F. Foulquier and T. Lefebvre, FASEB J, 2013, 27, 3478-3486. 


\section{LEGENDS TO THE FIGURES}

Figure 1. Effects of OGT and OGA inhibitors on protein O-GIcNAcylation. (A) A total cell extract of A431 cells was processed by SDS-PAGE and Western blots (WB) as described in Materials and Methods and probed with the anti-OGlcNAc antibodies CTD110.6 and RL2 as indicated. (B) A431 cells were incubated overnight in the absence (None) and presence of $20 \mu \mathrm{M}$ Thiamet $\mathrm{G}$ or $2 \mathrm{mM}$ BADGP as indicated. Whole cell lysates were processed by SDSPAGE and Western blots (WB) as described in Materials and Methods and probed with an anti-O-GICNAc antibody (RL2). The PVDF membranes were stripped and reprobed with an anti-GAPDH antibody as loading control. (C) The plot presents the mean \pm SEM $(n=4)$ protein O-GlcNAcylation from a set of experiments similar to the one shown in $A$ measuring the densitometry of the $O-$ GlcNAcylated bands corrected by loading as determined by protein staining with Fast Green. $\left(^{*}\right) \mathrm{p}<0.05$ as determined by the Student's t test. $(D)$ The plot presents the mean \pm SEM $(n=3)$ protein O-GICNAcylation from a set of experiments similar to the one shown in $A$ but using the indicated concentrations of Thiamet $G$ and measuring the densitometry of the $O$ GlcNAcylated bands corrected by loading as determined by protein staining with Fast Green.

Figure 2. Detection of O-GIcNAcylation signal in EGFR by immunoprecipitation and Western blot. $(A, B)$ The EGFR was immunoprecipitated (IP) from a cell extract of A431 cells using an anti-EGFR antibody and the immunocomplex was incubated in the absence (-) and presence $(+)$ of PNGase $F$ to remove $N$-glycans. The samples were immunoblotted (WB) with the anti-O-GlcNAc antibodies CTD110.6 (A) and RL2 $(B)$ as indicated. The PVDF membranes were stripped and reprobed with an anti-EGFR antibody as loading controls. Mock IP were performed using a nonrelevant IgG as negative controls. Upper and lower arrowheads point to the native and $N$-deglycosylated EGFR, respectively. (C) Immunoprecipitated (IP) EGFR, a mock immunoprecipitation using a non-relevant immunoglobulin G fraction (Mock IP) and an A431 total cell extract were immunoblotted (WB) using an anti-O-GlcNAc antibody (CTD110.6) in the absence (-) and presence 
(+) of an excess $(20 \mathrm{mM})$ of free GlcNAc as indicated to test the specificity of the antibody. The films were overexposed to ascertain absence of signal in the control with $20 \mathrm{mM}$ GlcNAc. Black arrowhead points to O-GlcNAcylated EGFR and gray arrowheads point to the heavy and light chains of lgG. The small lower inset shows protein staining with Fast Green as loading control. The arrowhead points to the EGFR. (D) A431 cells were incubated overnight in the absence (None) and presence of $20 \mu \mathrm{M}$ Thiamet G or $2 \mathrm{mM}$ BADGP as indicated. The samples were immunoprecipitated (IP) using an anti-O-GlcNAc antibody (RL2) and the immunocomplex processed by SDS-PAGE and Western blots (WB) as described in Materials and Methods and probed with anti-EGFR and anti-OGlcNAc (RL2) antibodies. The gray arrowheads point to the heavy and light chains of lgG. The heavy light chain of IgG was also stained with Fast Green as loading control. Mock IP was performed using a non-relevant IgG as negative control.

Figure 3. Effects of OGT and OGA inhibitors on EGFR O-GIcNAcylation signal. (A) A431 cells were incubated overnight in the absence (None) and presence of $20 \mu \mathrm{M}$ Thiamet $\mathrm{G}$ or $2 \mathrm{mM}$ BADGP as indicated. The samples were immunoprecipitated (IP) using an anti-EGFR antibody and the immunocomplex was incubated in the absence (-) and presence (+) of PNGase F to remove $N$ glycans and processed by SDS-PAGE and Western blots (WB) as described in Materials and Methods and probed with an anti-O-GIcNAc antibody (RL2). The PVDF membrane was stripped and reprobed with an anti-EGFR antibody as loading control. Upper and lower arrowheads point to the native and $N$ deglycosylated EGFR, respectively. $(B)$ The plot presents the mean \pm SEM $(n=$ 3) EGFR O-GICNAcylation from a set of experiments similar to the one shown in $A$ measuring the densitometry of the O-GlcNAcylated bands corrected by loading as determined by the total EGFR signal. $\left({ }^{*}\right) p<0.05$ as determined by the Student's t test.

Figure 4. Detection of O-GIcNAcylation signal in EGFR by immunoprecipitation and GIcNAz labeling. $(A)$ Serum-starved A431 cells were treated overnight with $40 \mu \mathrm{M}$ GlcNAz and incubated as indicated in the absence (-) and presence (+) of 10 nM EGF during $30 \mathrm{~min}$. Thereafter, the 
EGFR was immunoprecipitated (IP) and where indicated treated with PNGase F to remove $\mathrm{N}$-glycans. The samples were subjected to biotinylation and overlaid with streptavidin-HRP to detect GlcNAz-labeled proteins as described in Materials and Methods. Immunoprecipitated EGFR from cells non-treated with GlcNAz (No GlcNAz) and a mock IP are shown as negative controls. Protein staining of the immunoprecipitated EGFR with Fast Green is shown as loading control. Duplicate samples of the immunoprecipitated EGFR were processed by Western blot using an anti-EGFR antibody are shown where indicated. Upper and lower black arrowheads point to the native and $N$-deglycosylated EGFR, respectively, and the gray arrowhead points to a partially degraded $N$ deglycosylated EGFR. (B) Serum-starved A431 cells were treated in the absence (-) and presence (+) of $10 \mathrm{nM}$ EGFR for $30 \mathrm{~min}$. The reaction was arrested with $10 \%(\mathrm{w} / \mathrm{v})$ trichloroacetic acid, and the samples were processed for SDS-PAGE and immunoblot using an anti-phospho-tyrosine antibody to detect phosphorylated EGFR (P-EGFR) and an anti-EGFR antibody to detect total EGFR. GAPDH is shown as a loading control. $(C)$ The plot presents the mean \pm SEM $(n=3)$ of the densitometry of the EGFR/GAPDH signal ratio to control for possible loading differences. $\left({ }^{* *}\right) p<0.01$ as determined by the Student's t test. $(D)$ A431 cells were treated overnight with $1 \mu \mathrm{g} / \mathrm{ml}$ tunicamycin, the EGFR was immunoprecipitated (IP) and where indicated treated with PNGase $\mathrm{F}$ to remove residual $\mathrm{N}$-glycans. The samples were subjected to biotinylation and overlaid with streptavidin-HRP to detect GlcNAz-labeled proteins as described in Materials and Methods. Duplicate samples were probed with an anti-EGFR antibody. Immunoprecipitated EGFR from cells nontreated with GlcNAz (No GlcNAz) and a mock IP are shown as negative controls. Upper and lower arrowheads point to the native and $\mathrm{N}$-deglycosylated EGFR, respectively.

Figure 5. O-GIcNAcylation of EGFR in different cell lines. A431, A549, HeLa and EGFR-T17 cells were incubated overnight in the presence of $20 \mu \mathrm{M}$ Thiamet. The EGFR was immunoprecipitated (IP) using an anti-EGFR antibody and the immunocomplexes were processed by SDS-PAGE and immunoblot (WB) using anti-O-GlcNac (RL2) and anti-EGFR antibodies as described in Materials and Methods. A mock IP was performed using a non-relevant IgG as 
negative control.

Figure 6. Enhanced O-GICNAcylation of EGFR upon in vitro reaction catalyzed by immunoprecipitated OGT. (A) OGT and EGFR were independently immunoprecipitated from A431 cells and the samples were processed by immunoblot (WB) using anti-OGT and anti-EGFR antibodies to test for co-immunoprecipitation of both proteins. Mock IP were performed using a non-relevant IgG as negative controls. (B) OGT and EGFR were independently immunoprecipitated from A431 cells. Thereafter, the immunoprecipitated EGFR was incubated in the absence (-) and presence (+) of immunoprecipitated OGT and the O-GIcNAcylation reaction was performed upon addition of UDP-GIcNAc as described in Materials and Methods. The samples were immunoblotted (WB) with an anti-O-GlcNAc antibody (RL2) as indicated. OGT and EGFR were detected by WB using anti-OGT and antiEGFR antibodies, respectively. (C) The plot presents the mean \pm SEM EGFR O-GIcNAcylation in the absence (None) and presence of OGT from 3 independent experiments similar to the one shown in $B$ measuring the densitometry of the O-GICNAcylated EGFR band corrected by loading as determined by protein staining with Fast Green. $\left(^{*}\right) p<0.05$ as determined by the Student's t test.

\section{LEGEND TO THE GRAPH ABSTRACT}

The cartoon represents the EGFR at the plasma membrane where serine/threonine residues could be subjected to phosphorylation/dephosphorylation events by protein kinases (PK) and phospho-protein phosphatases (PPP) and to O-GlcNAcylation/deGlcNAcylation events by $O$-linked $\beta$ - $N$-acetylglucosamine transferase (OGT) and $O$-linked $\beta-N$ acetylglucosaminidase (OGA). 

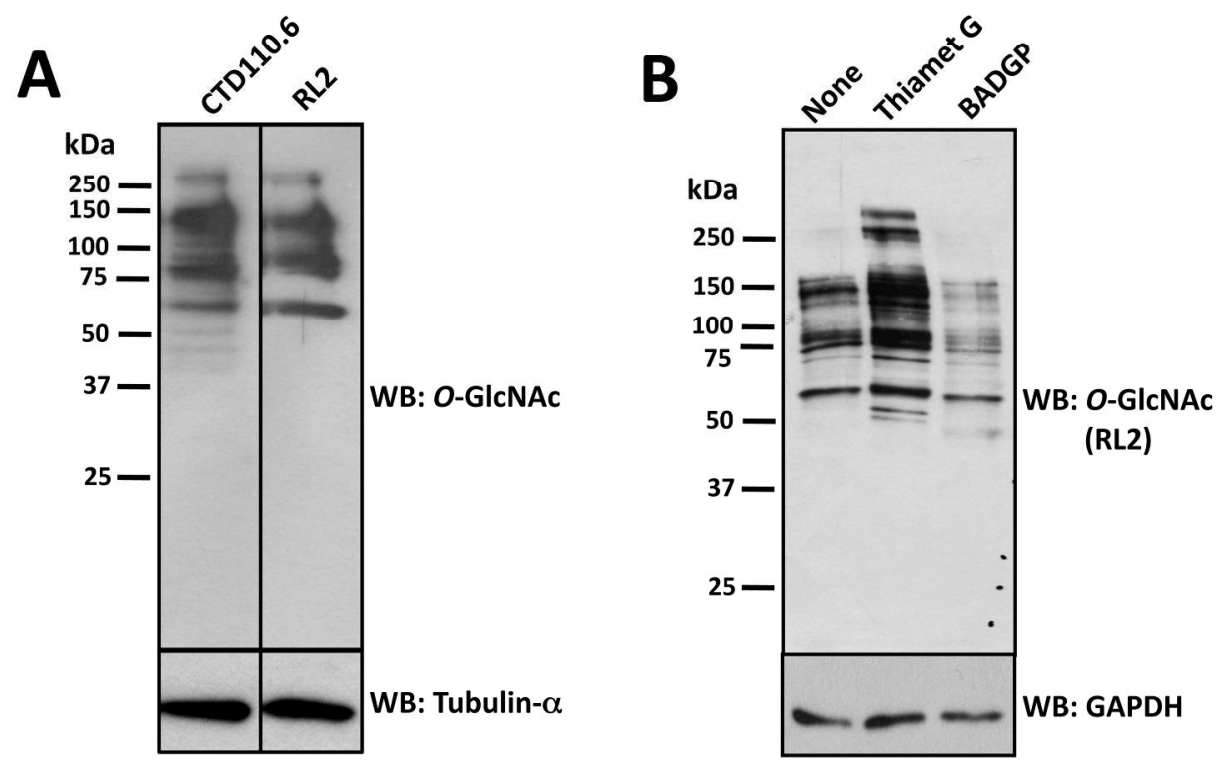

C

D
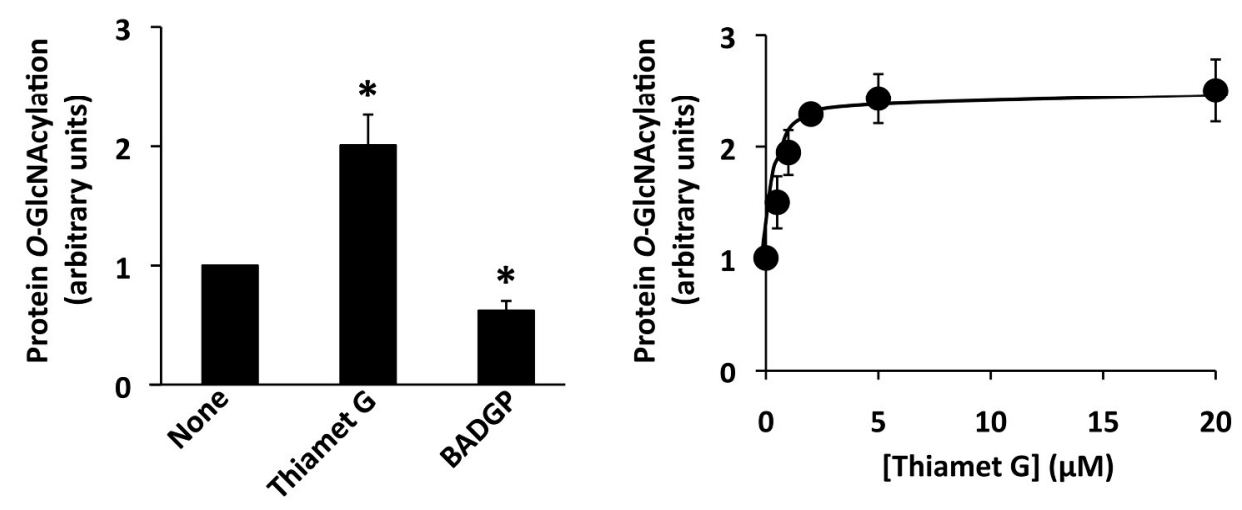
Fig. 2 (Stateva \& Villalobo, 2015)
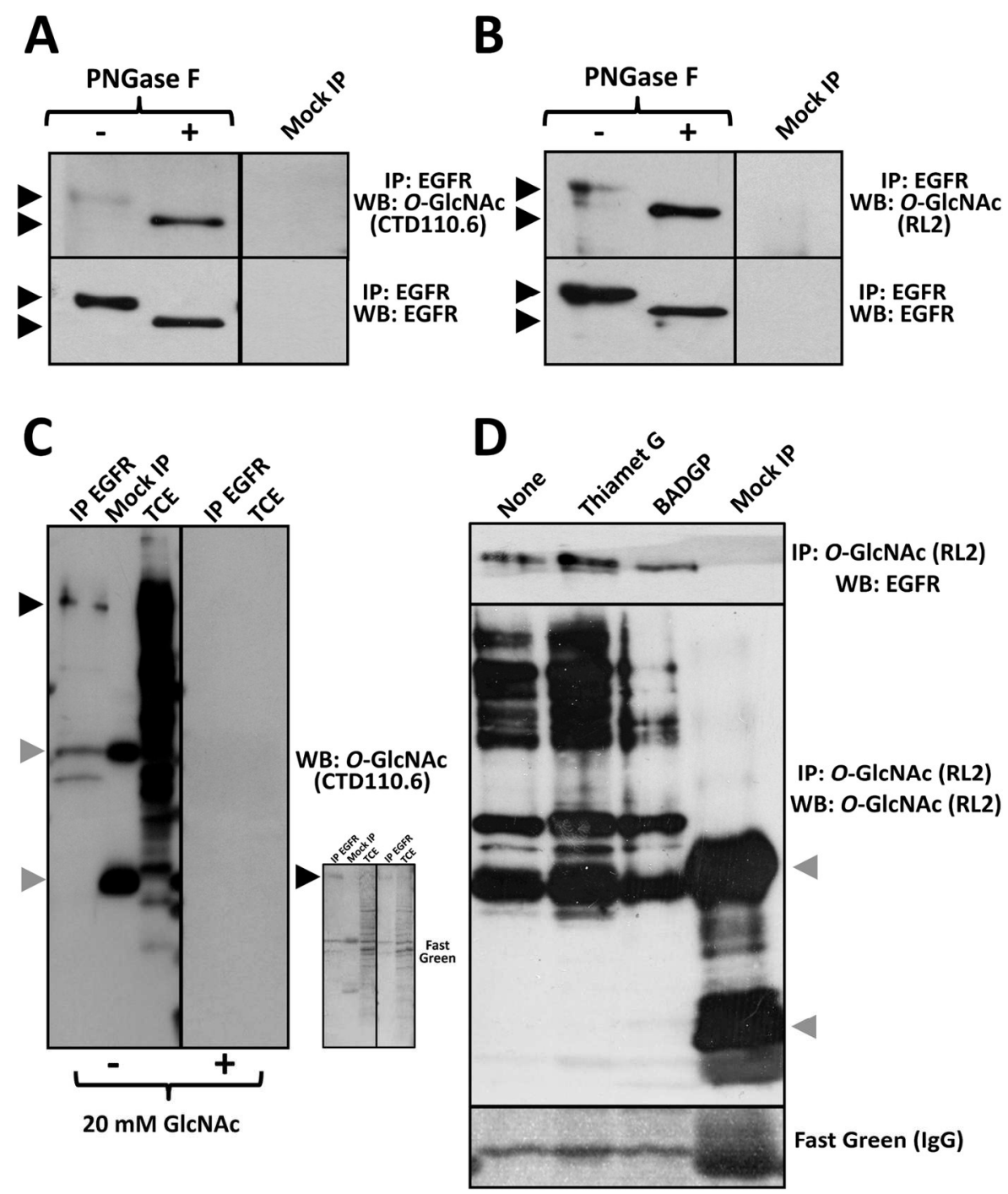

$148 \times 184 \mathrm{~mm}(300 \times 300 \mathrm{DPI})$ 


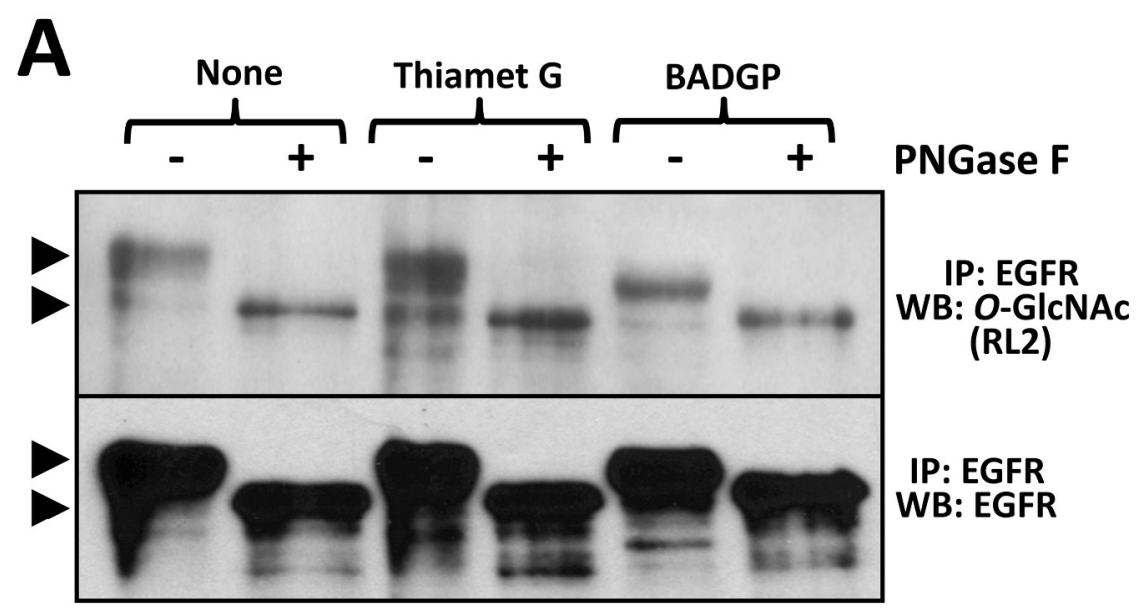

B

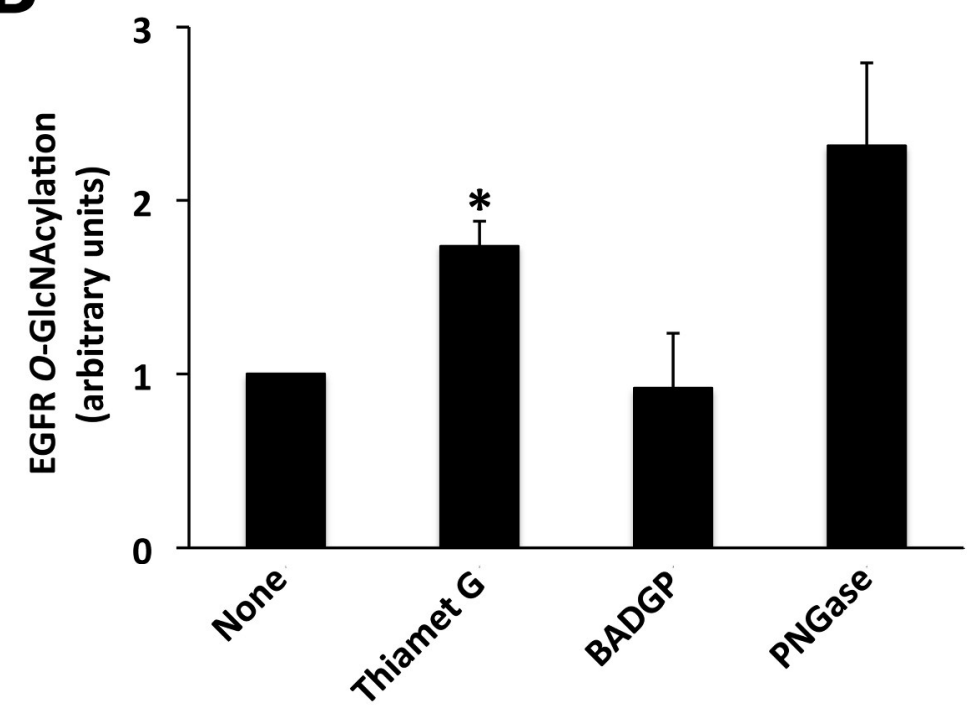

$194 \times 267 \mathrm{~mm}(300 \times 300$ DPI $)$ 


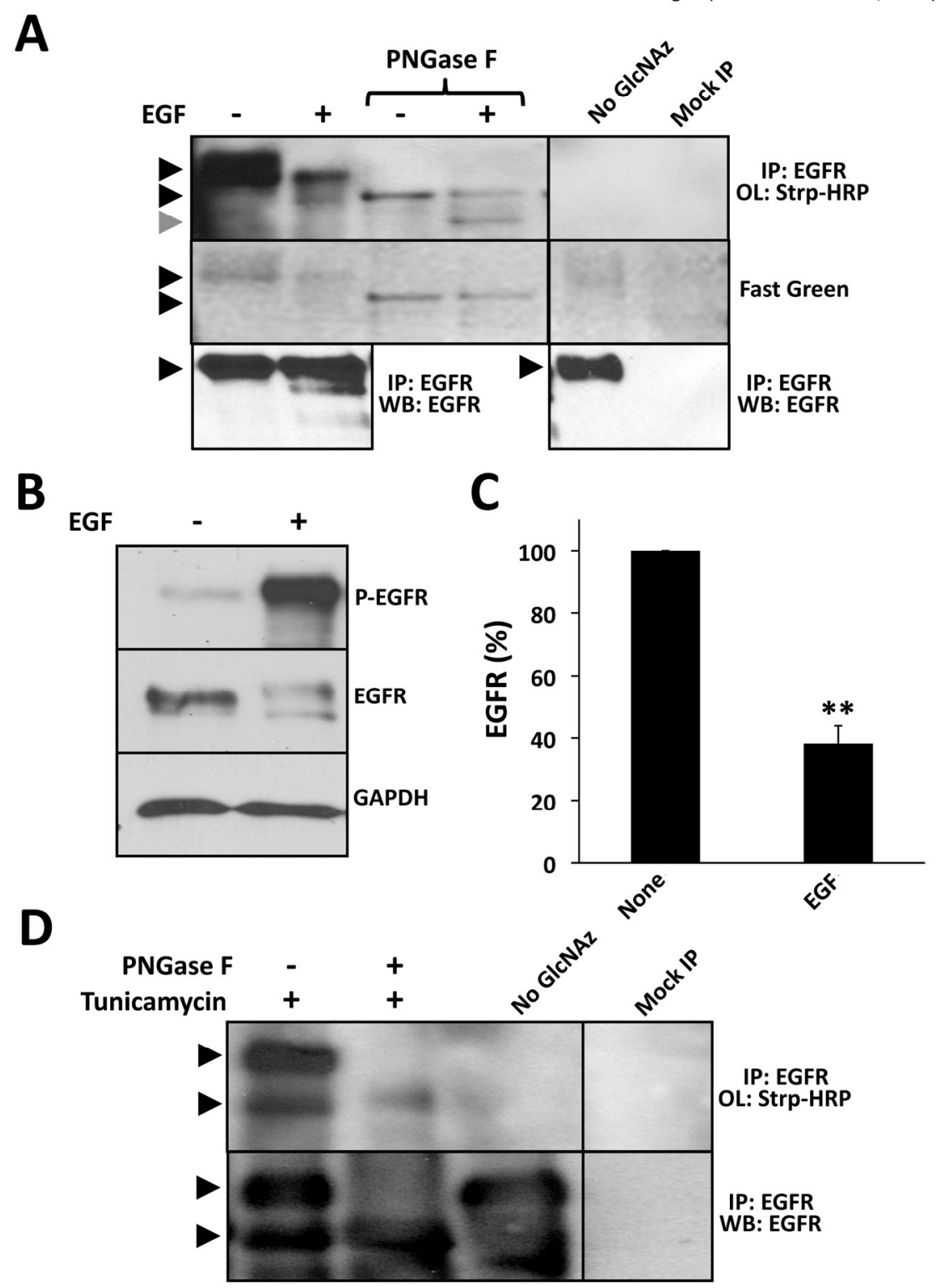

$156 \times 205 \mathrm{~mm}(300 \times 300$ DPI $)$ 
Fig. 5 (Stateva \& Villalobo, 2015)

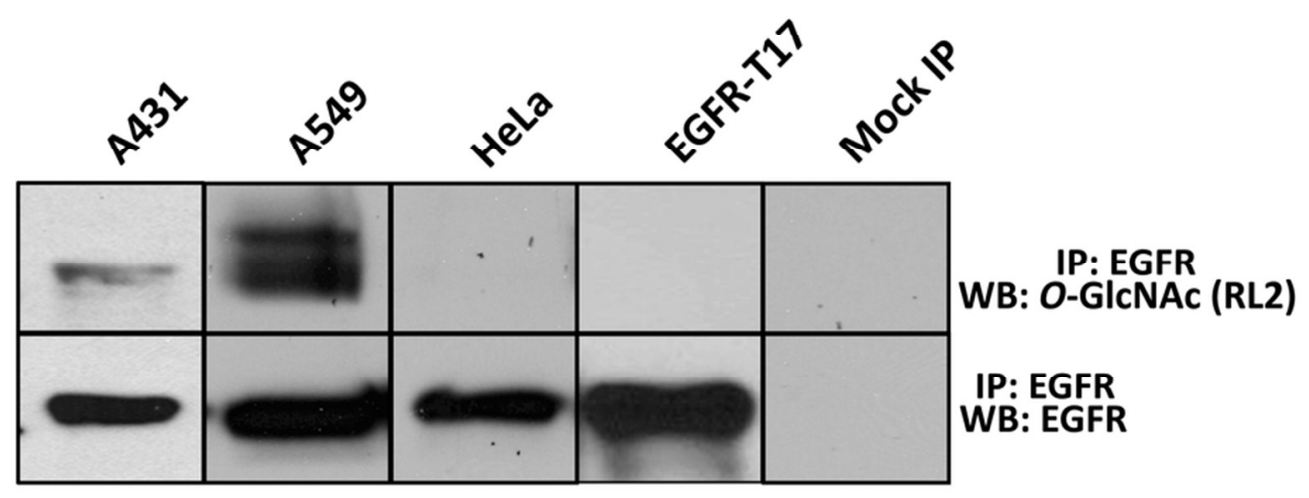

$78 \times 34 \mathrm{~mm}(300 \times 300$ DPI $)$ 
Fig. 6 (Stateva \& Villalobo, 2015)

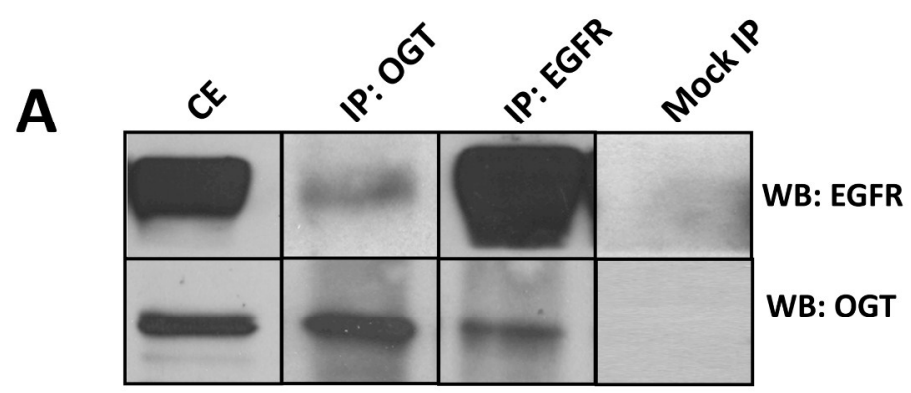

B

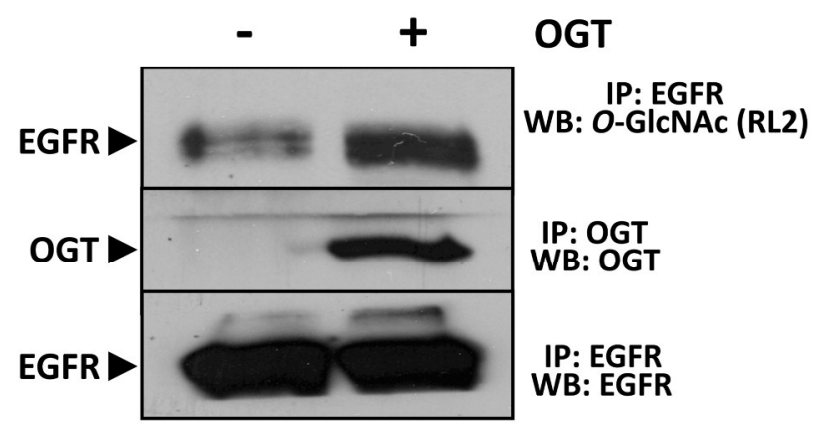

C

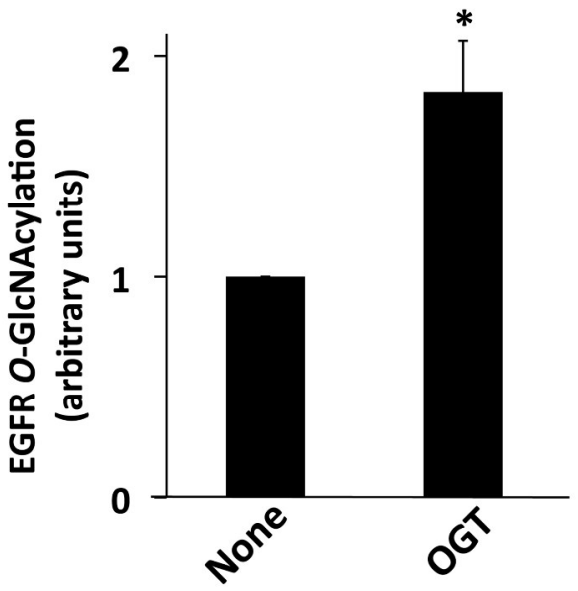

$186 \times 313 \mathrm{~mm}(300 \times 300$ DPI $)$ 